RESEARCH/Original article

\title{
Intercontinental hearing assessment - a study in tele-audiology
}

\author{
De Wet Swanepoel ${ }^{1,3}$, Dirk Koekemoer ${ }^{2}$ and Jackie Clark ${ }^{3,4}$
}

1 Department of Communication Pathology, University of Pretoria, South Africa

2 Research and Development Department, GeoAxon, South Africa

3 Callier Center for Communication Disorders, University of Texas at Dallas, USA

4 Department of Speech and Hearing Therapy, University of the Witwatersrand, South Africa

Correspondence:

Dr De Wet Swanepoel,

Department of Communication Pathology,

University of Pretoria,

Pretoria 0002,

South Africa

(Fax: +27 12420 3517; Email: dewet.swanepoel@up.ac.za)

\begin{abstract}
Summary
We evaluated the validity of remote pure-tone audiometric testing conducted from North America on subjects in South Africa. Desktop-sharing computer software was used to control the audiometer in Pretoria from Dallas, and PC-based videoconferencing was employed for clinician and subject communication. Thirty adult subjects were assessed, comparing the pure tone audiometric thresholds $(125-8000 \mathrm{~Hz})$ obtained through conventional face-to-face and remote testing. Face-to-face and remote audiometry thresholds differed by $10 \mathrm{~dB}$ in only $4 \%$ of cases overall. The limits of agreement between the two techniques were -8 and $7 \mathrm{~dB}$ with a $90 \%$ confidence interval of -5 to $5 \mathrm{~dB}$. The average reaction times to stimulus presentations were similar, within -108 and $121 \mathrm{~ms}$. The average test duration was $21 \%$ longer for remote testing (10.4 vs. $8.2 \mathrm{~min}$ ). There were no clinically significant differences between the results obtained by remote intercontinental audiometric testing and conventional face-to-face audiometry. It may therefore be possible to expand the reach of audiological services into remote underserved regions of the world.
\end{abstract}




\section{Introduction}

Hearing loss is the most common chronic disabling condition globally and in 2005 was estimated to affect 642 million people to some degree.[1,2] A range of interventions can reduce the consequences of hearing loss,[1] but the basis for intervention is early identification and accurate diagnosis.

Except in the case of very young infants or other difficult-to-test populations (e.g. people who are severely handicapped) who cannot provide reliable behavioural responses, the gold standard for describing hearing sensitivity is pure tone audiometry. This procedure determines behavioural thresholds to pure tone acoustic signals of various frequencies (i.e. 250, 500, 1000, 2000, 4000 and $8000 \mathrm{~Hz}$ ). Thresholds represent the lowest intensity where the patient responds to the acoustic signal providing information on hearing sensitivity (i.e. degree and configuration of hearing loss). When combined with bone conduction testing, in which the cochlea is stimulated directly by presenting sound vibrations to the forehead or mastoid, the type of hearing loss can also be distinguished. The findings of this test procedure form the basis for clinical decisions regarding audiological treatment, referrals and intervention.

Unfortunately these diagnostic test procedures are not available to the majority of those with hearing loss. The reasons include a shortage of staff trained to conduct audiometric tests and limited infrastructure such as the audiometers and sound booths necessary for diagnostic pure tone testing.[3,4] Telemedicine offers the possibility of extending hearing assessments to underserved communities. New generation audiometers are portable, highly flexible devices that are compatible with telemedicine and some include features that could reduce the need for expensive sound booths for testing. Such features include live monitoring of environmental noise, double sound-attenuation with insert and circumaural earphones, and active noise-cancellation.[5]

Initial reports of pure tone audiometry conducted via telemedicine have revealed promising results, which indicate essentially equivalent findings when compared to conventional face-toface testing.[6-9] However, the initial studies have been limited in the distance at which the remote testing was conducted (between 1 and $1100 \mathrm{~km}$ ). Cross-continent or transatlantic telemedicine hearing assessments have not been reported.

Healthcare professionals in industrialized countries (i.e. the US) may be able to provide hearing healthcare services via telemedicine in countries where no such professionals are available (i.e. most countries in Africa). In underserved areas like Africa, Internet connectivity is becoming increasingly available although it may have to rely on mobile phone networks.[5] The present investigation therefore utilized a telemedicine compliant computer-based audiometer, connected to the Internet through a mobile phone network, to determine the validity of synchronous pure tone audiometry conducted from North America on subjects in sub-Saharan Africa. 


\section{Methods}

The study was approved by the appropriate ethics committee. Thirty subjects (with 60 ears) between 18 and 65 years of age were recruited for the study (18 women and 12 men). The subjects were volunteers living in Pretoria. Most volunteers (80\%) had hearing thresholds that all fell within the range commonly specified as normal hearing $(\leq 25 \mathrm{~dB})$. According to more recently proposed criteria [10] for normal hearing thresholds $(\leq 15 \mathrm{~dB}), 50 \%$ of the volunteers had normal hearing across all frequencies.

\section{Equipment}

A portable audiometer with insert earphones (KUDUwave 5000, GeoAxon, South Africa) was used to measure pure tone air conduction thresholds. The audiometer is software controlled and connected to a netbook computer (Acer Aspire One PC, Windows XP) via a USB port and to the Internet through a 3G mobile modem. The audiometer's circumaural earphones are placed over insert earphones to increase the attenuation of environmental sound. Environmental noise levels are monitored by the device with an external and internal microphone to measure noise levels for test compliance.

At the test site, a physician acted as facilitator to ensure that the audiometer software operated properly and that the insert earphone probes and circumaural earphones were placed correctly on the subject's ears. A $3 G$ cellular network was used to connect to the Internet at the test site in South Africa and a broadband wireless or LAN network was used to connect to the Internet at the remote clinician site in the US. The computers at both sites were configured with videoconferencing software (Skype Video call version 4, Luxembourg) and hardware (microphone and webcam). Remote computing was performed through application sharing software (TeamViewer 4, Göppingen, Germany) so that the clinician in Dallas could control the computer in Pretoria to facilitate the remote testing (Figure 1). Information privacy was ensured through a unique identification number and password sent from the computer at the test site to the computer at the remote site through Skype. All the information collected was stored on the computer in Pretoria. The remote clinician was in audiovisual contact with the patient through the videoconference.

\section{Measurements}

All subjects were tested with pure tone air conduction audiometry in both ears across octaveinterval frequencies from 125 to $8000 \mathrm{~Hz}$ in two test configurations scheduled in the same sound treated room. In conventional mode the clinicians tested the subject's hearing face-to-face. In telemedicine mode the subject was tested in the same room but by a clinician 14,680 km away in Dallas. The remote testing was facilitated through desktop application-sharing and interactive videoconferencing software (Figure 1). During the remote testing, an on-site physician positioned the earphones for testing and the remote clinician instructed patients regarding the test procedure. The test order (face-to-face first, or remote first) was alternated and clinicians were kept blind to each other's results. The local clinician was either outside the test room or seated at the back of the computer screen during the remote testing. The remote clinician saved data once the test was completed and notified the local clinician via the videoconference. All testing (one face-to-face and one remote test) was conducted on the same day.

The test protocols and procedure were identical between local and remote clinicians. A conventional $10 \mathrm{~dB}$ down and $5 \mathrm{~dB}$ up bracketing method was used to determine hearing 
thresholds. The frequency testing started at $1000 \mathrm{~Hz}$ and proceeded to higher frequencies. After testing at $8000 \mathrm{~Hz}$ the lower frequencies were evaluated, starting at $500 \mathrm{~Hz}$ and reducing to 125 $\mathrm{Hz}$. Subjects indicated responses to the stimulus by using a hand-held response switch. When pressed, a visual response was indicated on the computer screen and the reaction time was documented. The subject's responses appeared on the audiogram and were colour-coded: a green mark indicated that the response fell within the allowed time frame and a yellow mark indicated that the response fell outside the allowed timeframe or that there was no response to the stimulus presentation.

The average reaction time from stimulus presentation to the subject pressing the response button was recorded in a sub-group of 9 patients to compare the reaction times between the face-to-face and remote audiometric assessment conditions.

Recording time was also documented for conducting the conventional and remote audiometric tests on 22 of the subjects. The recording was initiated with the first stimulus presentation to the first ear tested and was terminated after the last patient response was recorded for the last ear tested.

\section{Data analysis}

Limits of agreement (mean difference \pm 2 SD) between the two techniques, and $90 \%$ confidence intervals, were determined according to the method of Bland \& Altman [11] for assessing agreement between two methods. A t-test across frequencies $(125-8000 \mathrm{~Hz})$ was conducted to determine if there was a significant difference between the results obtained with the conventional face-to-face and remote test conditions.

\section{Results}

The average thresholds observed during face-to-face (FTF) and remote (RT) audiometry are shown in Table 1. The average absolute difference between thresholds was $2.4 \mathrm{~dB}$ (SD 2.9) and varied between 2 and $2.9 \mathrm{~dB}$ across frequencies. There were no significant differences between test conditions across frequencies $(P>0.05)$.

Face-to-face and remote audiometry thresholds differed by $10 \mathrm{~dB}$ in only $4 \%$ of cases overall, see Figure 2. The limits of agreement between the two techniques (FTF-RT) were -8 and $7 \mathrm{~dB}$ with a $90 \%$ confidence interval of -5 to $5 \mathrm{~dB}$. Figure 3 illustrates the difference between face-to-face and remote audiometry thresholds for two of the seven octave frequencies assessed.

A separate analysis of correspondence between face-to-face and remote audiometry was conducted, for thresholds outside the range of normal hearing $(\leq 15 \mathrm{~dB})$, representing $13 \%$ of all thresholds (54 of 420 threshold comparisons). Correspondence was within $5 \mathrm{~dB}$ or less in $98 \%$ of comparisons. The remaining normal hearing thresholds $(\leq 15 \mathrm{~dB})$, representing $87 \%$ of all thresholds, corresponded within $5 \mathrm{~dB}$ or less in $95 \%$ of cases.

The average reaction time from stimulus presentation to response is shown in Table 2, as recorded in 9 of the 30 subjects for all threshold measurements in both ears. The mean values for the average reaction times differed by less than $2 \mathrm{~ms}$. The average difference between the average reaction times with face-to-face and remote audiometry was 2 ms (ranging between -108 and 121 
$\mathrm{ms}$ ) and the limits of agreement between the two techniques (FTF-RT) were -135 and 139 ms with a $90 \%$ confidence interval of -96 to $97 \mathrm{~ms}$.

The time required to complete an audiogram for both ears of a subject was recorded for 22 of the 30 subjects. The average time for the conventional face-to-face test was 8.2 min (SD 2.1) compared to 10.4 min (SD 2.1) for remote testing, which is a $21 \%$ longer average test duration.

\section{Discussion}

Apart from an anecdotal report of a patient in the USA being tested from Brazil [12], the longest distance for remote synchronous audiometric threshold testing previously reported in a group study was $1100 \mathrm{~km}$ and was within the same country.[9] The present study demonstrates the feasibility of remote audiometric testing from North America to Africa. The intercontinental assessment revealed no significant difference between pure tone hearing thresholds when compared to conventional face-to-face testing. The average reaction times recorded with face-toface compared to remote testing were similar, within -108 and $121 \mathrm{~ms}$, but the average test duration was slightly longer for remote-testing although it varied with a $90 \%$ confidence interval of -0.5 and 5.9 minutes. This may have been due to delays caused by the mobile phone network Internet connection, but did not have any effect on the test results.

The results of the present study are in close agreement with previous reports comparing pure tone audiometry thresholds obtained remotely and conventional face-to-face tests.[6-9] The first studies were conducted in the USA by Givens and colleagues.[6,7] The mean threshold differences between the remote and conventional test procedures employed by these authors varied between 0.0 and $1.3 \mathrm{~dB}$ across frequencies (250 to $8000 \mathrm{~Hz}$ ) compared to differences of between -1 and $0.1 \mathrm{~dB}$ across frequencies $(125$ to $8000 \mathrm{~Hz})$ in the current study. In another report by Choi and colleagues [8] the remote versus conventional pure tone hearing thresholds varied by less than $5 \mathrm{~dB}$ in $89 \%$ of threshold comparisons compared to $96 \%$ in the present study. A similar report for 30 normal hearing subjects tested with pure tone audiometry in a remote and a face-toface configuration, revealed threshold correspondence within $5 \mathrm{~dB}$ in $97 \%$ of cases compared to $96 \%$ in the present study.[9]

The variability observed in threshold correspondence within $5 \mathrm{~dB}$ (90\% confidence interval of -5 to $5 \mathrm{~dB}$ ) for remote and face-to-face audiometry corresponds to normal clinical variability (testretest variability) in determining hearing thresholds with pure tone audiometry. Typical test-retest variability in pure tone audiometry is $\pm 5 \mathrm{~dB}$ in individual subjects, whether children or adults.[13,14] The investigations by Choi and colleagues [8] and Krumm and colleagues also included an evaluation of test-retest reliability for conventional face-to-face audiometry.[9] Although the test-retest variability was reported as slightly less than that of remote compared to face-to-face thresholds, there was no significant difference between either comparison.

Although the majority of comparisons (87\%) in the present study were for normal hearing thresholds $(\leq 15 \mathrm{~dB})$, some $(n=54)$ elevated thresholds $(\geq 20 \mathrm{~dB})$ which indicate hearing loss were also included. The correspondence for these elevated thresholds obtained with face-to-face and remote test conditions was very close, with $98 \%$ within $5 \mathrm{~dB}$ or less of each other. This was slightly better than the correspondence for the remaining normal hearing $(\leq 15 \mathrm{~dB})$ thresholds 
(95\%). Validation in more cases of hearing loss, especially with more severe degrees of hearing loss must, however, still be performed.

The present threshold findings for remote intercontinental audiometric testing indicate that there is no clinically significant difference when compared to conventional face-to-face audiometry. The possibility of testing hearing accurately at long distance opens the possibility of expanding the reach of audiological services into remote underserved regions of the world. Although much work remains to be done, including monitoring remote sites for compliance and determining patient and clinician acceptance of such tests, the initial findings appear very promising.

\section{References}

1. World Health Organization. Primary ear and hearing care training manuals. Geneva 2006 See: http://www.who.int/pbd/deafness/activities/hearing_care/en/index.html (last checked 17 November 2009)

2. World Health Organization. The global burden of disease: 2004 update. Geneva 2008 See: http://www.who.int/healthinfo/global_burden_disease/GBD_report_2004update_full.pdf (last checked 17 November 2009)

3. Fagan JJ, Jacobs M. Survey of ENT services in Africa: Need for a comprehensive intervention. Global Health Action 2009 See: http://www.globalhealthaction.net/index.php/gha/article/viewArticle/1932 (last checked 17 November 2009)

4. Goulios H, Patuzzi RB. Audiology education and practice from an international perspective. Int J Audiol 2008; 47: 647-64

5. Swanepoel, D., Olusanya, B. O. \& Mars, M. 2009. Hearing healthcare delivery in sub-Saharan Africa - a role for tele-audiology. $J$ Telemed Telecare, In Press

6. Givens GD, Blanarovich A, Murphy T, Simmons S, Blach D, Elangovan S. Internet-based tele-audiometry system for the assessment of hearing: a pilot study. Telemed $J$ E Health 2003; 9: 375-8

7. Givens GD, Elangovan S. Internet application to tele-audiology--"nothin' but net". Am J Audiol 2003; 12: 59-65

8. Choi JM, Lee HB, Park CS, Oh SH, Park KS. PC-based tele-audiometry. Telemed J E Health 2007; 13: 501-8

9. Krumm M, Ribera J, Klich R. Providing basic hearing tests using remote computing ttechnology. J Telemed Telecare 2007; 13: 406-10

10. Martin FN, Champlin CA. Reconsidering the limits of normal hearing. J Am Acad Audiol 2000 Feb; 11: 64-6

11. Bland JM, Altman DG. Statistical methods for assessing agreement between two methods of clinical measurement. Lancet 1986; 1: 307-10

12. Krumm M. Audiology telemedicine. J Telemed Telecare 2007;13: 224-9

13. Stuart A, Stenstrom R, Tompkins C, Vandenhoff S. Test-retest variability in audiometric threshold with supraaural and insert earphones among children and adults. Audiology 1991; 30: 82-90

14. Smith-Olinde L, Nicholson N, Chivers C, Highley P, Williams DK. Test-retest reliability of in situ unaided thresholds in adults. Am J Audiol 2006; 15: 75-80 
Table 1. Average thresholds for face-to-face (FTF) and remote (RT) audiometry

\begin{tabular}{|l|c|c|c|c|c|c|c|c|}
\hline & $\mathbf{1 2 5} \mathbf{~ H z}$ & $\mathbf{2 5 0} \mathbf{~ H z}$ & $\mathbf{5 0 0 ~} \mathbf{~ H z}$ & $\begin{array}{c}\mathbf{1 0 0 0} \\
\mathbf{H z}\end{array}$ & $\begin{array}{c}\mathbf{2 0 0 0} \\
\mathbf{H z}\end{array}$ & $\begin{array}{c}\mathbf{4 0 0 0} \\
\mathbf{H z}\end{array}$ & $\mathbf{8 0 0 0} \mathbf{~ H z}$ & $\begin{array}{c}\text { Overal } \\
\mathbf{l}\end{array}$ \\
\hline $\begin{array}{l}\text { Mean FTF threshold } \\
\text { (SD) }\end{array}$ & $9.2(8.8)$ & $\begin{array}{c}7.6 \\
(7.7)\end{array}$ & $\begin{array}{c}8.6 \\
(8.7)\end{array}$ & $\begin{array}{c}8.7 \\
(10.5)\end{array}$ & $\begin{array}{c}8.5 \\
(8.7)\end{array}$ & $\begin{array}{c}5.3 \\
(10.5)\end{array}$ & $\begin{array}{c}10.1 \\
(17.1)\end{array}$ & $\begin{array}{c}8.3 \\
(10.7)\end{array}$ \\
\hline $\begin{array}{l}\text { Mean RT threshold } \\
\text { (SD) }\end{array}$ & $10.2(8.9)$ & $\begin{array}{c}8.1 \\
(8.5)\end{array}$ & $\begin{array}{c}9.3 \\
(8.5)\end{array}$ & $\begin{array}{c}8.9 \\
(10.5)\end{array}$ & $\begin{array}{c}8.4 \\
(8.1)\end{array}$ & $\begin{array}{c}6.1 \\
(10.4)\end{array}$ & $\begin{array}{c}10.9 \\
(17.0)\end{array}$ & $\begin{array}{c}8.8 \\
(10.7)\end{array}$ \\
\hline
\end{tabular}

Table 2. Average reaction times (ms) and number of presentations for face-to-face and remote audiometry (9 subjects)

\begin{tabular}{|l|c|c|c|}
\hline & Mean & SD & No of presentations \\
\hline Face-to-face audiometry & 596 & 191 & 93 \\
\hline Remote audiometry & 594 & 189 & 75 \\
\hline Mean difference (FTF-RT) & 2 & 69 & 18 \\
\hline
\end{tabular}

\section{Figure legends}

1. Remote audiometric test configuration with interactive videoconferencing. The clinician is visualized in the top right corner with the subject being evaluated visualized in the bottom right corner. The left and right ear thresholds are visualized on the audiogram on the left side of the figure (There is a video recording of a test session from Dallas to South Africa at: http://www.youtube.com/watch?v=HDfjuvP0Dh0).

2. Distribution of threshold differences between face-to-face and remote audiometry (420 threshold comparisons). $0 \mathrm{~dB}$ represents perfect correspondence; $\pm 5 \mathrm{~dB}$ represents normal testretest variability. 
3. Difference between thresholds measured in face-to-face and remote audiometry (60 comparisons) versus the average of the two measurements. (a) at $125 \mathrm{~Hz}$ and (b) at $8 \mathrm{kHz}$

Fig 2

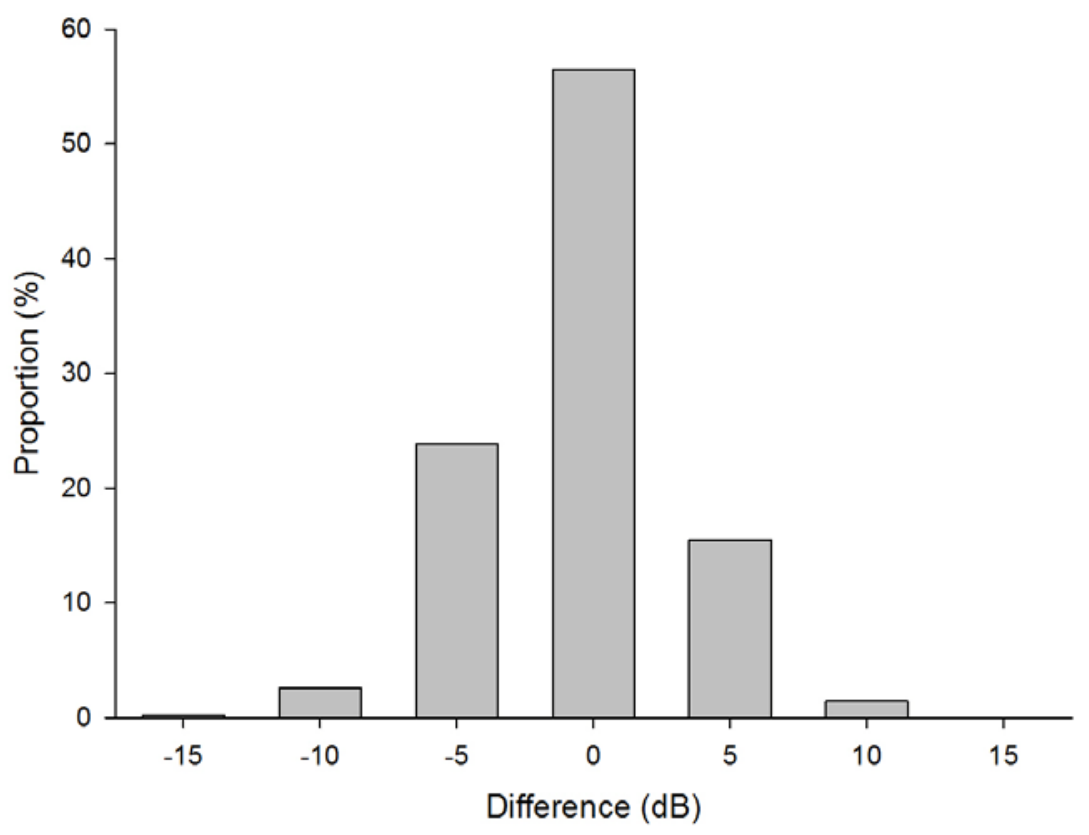


Fig $3-125 \mathrm{~Hz}$

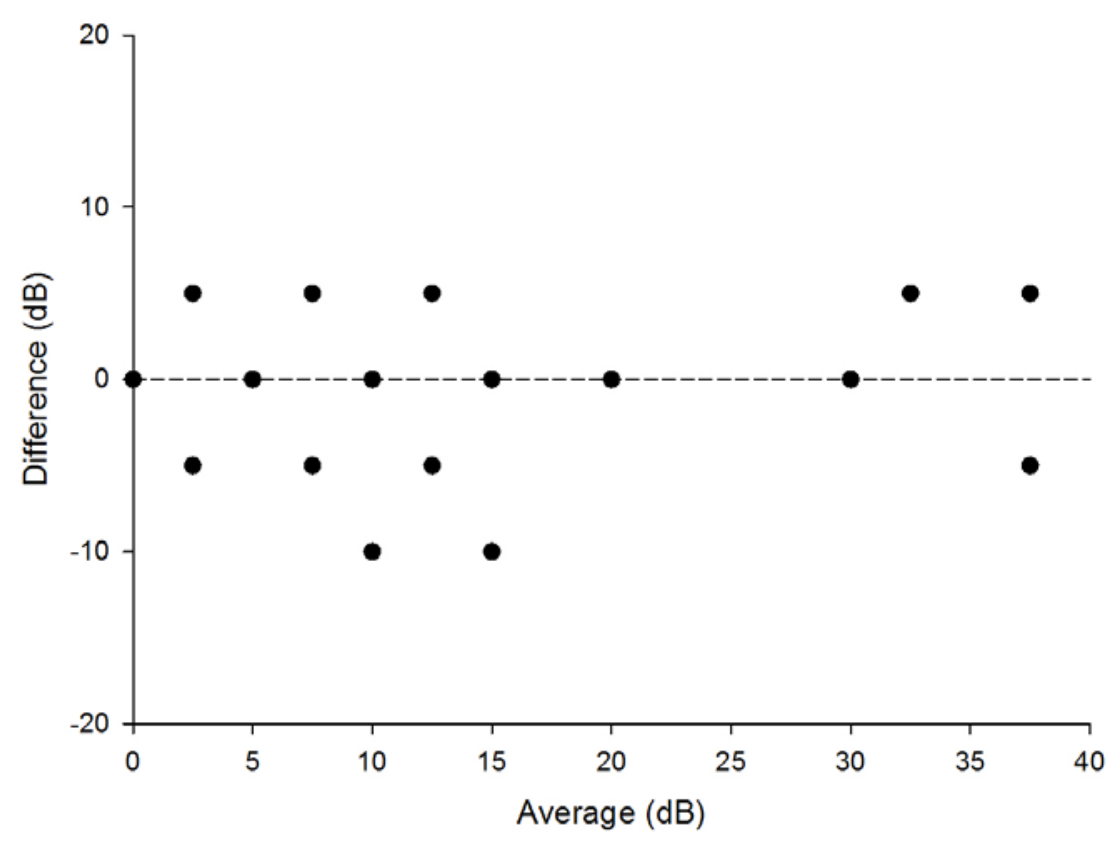


Fig $3--8$ kHz



